\title{
TWO CLASSES OF LOCALLY COMPACT SOBER SPACES
}

\author{
KARIM BELAID, OTHMAN ECHI, AND RIYADH GARGOURI \\ Received 15 December 2004 and in revised form 6 July 2005
}

We deal with two classes of locally compact sober spaces, namely, the class of locally spectral coherent spaces and the class of spaces in which every point has a closed spectral neighborhood (CSN-spaces, for short). We prove that locally spectral coherent spaces are precisely the coherent sober spaces with a basis of compact open sets. We also prove that CSN-spaces are exactly the locally spectral coherent spaces in which every compact open set has a compact closure.

\section{Introduction}

A sober space is a $T_{0}$-space in which every closed irreducible subset is a singleton closure. Every sober space $X$ can be recovered from the frame (complete distributive Brouwerian lattice) $O(X)$ of open sets as the prime spectrum $\operatorname{Spec}(O(X))$ and each $T_{0}$-space $Y$ can be universally embedded into the sober space $\operatorname{Spec}(O(Y))$.

A space will be called compact if every open cover has a finite subcover, and locally compact if every neighborhood of a point contains a compact neighborhood. The theory of locally compact sober spaces is completely captured by the theory of continuous frames (continuous distributive lattices), because for each locally compact sober space $X$, the lattice $\mathcal{O}(X)$ is a continuous frame such that $X \cong \operatorname{Spec}(\mathcal{O}(X))$ and every continuous frame $L$ has a locally compact sober space $\operatorname{Spec}(L)$ as its prime spectrum such that $L \cong \mathcal{O}(\operatorname{Spec}(L))$. A locally compact sober space has a basis of compact open sets if and only if its frame $O(X)$ of open sets is algebraic [1, page 115], and the spectrum of every algebraic frame is a locally compact sober space with a basis of compact open sets. A space $X$ whose frame $O(X)$ is algebraic is sometimes called a quasi-Boolean space [4].

Let $X$ be a topological space and $A$ a subset of $X$. The saturation of $A$ is

$$
\operatorname{Sat}(A)=\cap\{U \mid A \subseteq U \text { and } U \text { is open in } X\} .
$$

One says that $A$ is saturated, if $A=\operatorname{Sat}(A)$. In $T_{0}$-spaces the right classes of compact subsets are the saturated ones. A subset is compact if and only if its saturation is compact. A space is called coherent if the intersection of two saturated compact sets is compact. 
A space is called stably compact if it is compact, locally compact, coherent, and sober. One of the principal results of the theory of stably compact spaces [2, page 474] is that a $T_{0^{-}}$ space is stably compact if and only if the patch topology is a compact Hausdorff topology. The patch topology is an invention of Hochster; a basis of its open sets consists of the given open sets plus the complements of the compact saturated sets. Stably compact spaces and partially ordered compact Hausdorff spaces are practically one and the same thing [2, page 482]. The frame $O(X)$ of a stably compact space is a stably continuous frame (i.e., has a multiplicative way-below relation) with compact top element; conversely, a stably continuous frame with compact maximal element has a stably compact spectrum. Thus stably compact spaces are again practically the same as stably continuous frames with compact top element [2, page 489].

If $R$ is a commutative ring with an identity, then its prime ideal spectrum is the prime spectrum of the lattice $L$ of radical ideals and that is an algebraic frame and thus $\operatorname{Spec}(R)$ is a locally compact sober space with a basis of compact open sets (and due to the presence of an identity in $R$ ) it is compact. It is Hochster's merit to have shown [3] that every compact, locally compact, coherent, sober space with a basis of compact open sets arises in this way. One says that a space is spectral, if it is a compact, locally compact, coherent, sober space with a basis of compact open sets [3].

The primitive spectrum of a $C^{*}$-algebra is a locally compact Baire space (although if the algebra is not separable, it may fail to be sober); so locally compact spaces receive a considerable interest in the operator algebra community.

Due to the fact that authors in theoretical computer science dealing with the denotational semantics of programming languages have been interested in $T_{0}$ spaces and continuous lattices since Scott's article [5], there is considerable interest in this topic.

The role of compact, locally compact, and sober spaces with a basis of compact open sets in Stone's theorem on the representation of distributive lattices and similar contexts was discussed in [4] in 1972.

Hochster [3] has called a space locally spectral if it has a cover by open spectral subspaces.

Here we are interested in the study of two classes of locally compact sober spaces, namely, the class of locally spectral coherent spaces and the class of spaces in which every point has a closed spectral neighborhood (CSN-spaces, for short).

This paper contributes two "local-global" results, the first one explaining that locally spectral coherent spaces are precisely the coherent sober spaces with a basis of compact open sets, and the second that CSN-spaces are exactly the locally spectral coherent spaces in which every compact open set has a compact closure.

\section{Locally spectral coherent spaces}

This section deals with an intrinsic topological characterization of locally spectral coherent spaces.

First, recall some characterizations M. Hochster.

Theorem 2.1 [3]. The locally spectral spaces are precisely the underlying spaces of preschemes. 
Theorem 2.2 [3]. The following conditions on a topological space $X$ are equivalent:

(i) $X$ is a coherent locally spectral;

(ii) $X$ is the underlying space of an open subscheme of an affine scheme;

(iii) $X$ is the underlying space of some scheme;

(iv) $X$ is homeomorphic with an open subspace of a spectral space.

First, we need two lemmas, the first one is cited in [1, Proposition 7, page 122].

LEMma 2.3. Let $X$ be a topological space and $U$ a nonempty open set of $X$. Then the mapping $V \mapsto \bar{V}$ defines a bijection from the set of irreducible nonempty closed subsets of $U$ onto the set of irreducible nonempty closed subsets of $X$ meeting $U$. The inverse bijection is $Z \mapsto$ $Z \cap U$.

The second lemma is a special case of [2, Exercise O-5.15(ii)].

Lemma 2.4. Let $X$ be a sober space and $U$ a nonempty open set of $X$. Then $U$ is sober.

Theorem 2.5. The following conditions on a topological space $X$ are equivalent:

(i) $X$ is a locally spectral coherent space;

(ii) there exists a spectral space $Y$ with a unique closed point $\omega$ such that $X$ is homeomorphic with the subspace $Y \backslash\{\omega\}$ of $Y$;

(iii) $X$ is a coherent sober space with a basis of compact open sets;

(iv) $X$ is an open subset of a spectral space.

Proof. As (i) $\Leftrightarrow$ (iv) according to Theorem 2.2, it suffices to prove the equivalence between conditions (ii), (iii), and (iv).

(ii) $\Rightarrow$ (iii). Let $(Y, \mathscr{T})$ be a spectral space with a unique closed point $\omega$.

(a) Since $Y$ is the unique open set of $Y$ containing $\omega$, the topology of $X$ is $\{U \in \mathscr{T}$ | $\omega \notin U\}$. Hence $X$ is coherent with a basis of compact open sets.

(b) By Lemma 2.4, the open subset $X$ of the sober space $Y$ is also sober.

(iii) $\Rightarrow$ (ii) and (iv).

(c) Let $\omega \notin X$ and $\tilde{X}=X \cup\{\omega\}$. Consider the topology $\tilde{\mathscr{T}}=\mathscr{T} \cup\{\tilde{X}\}$ on $\tilde{X}$. It is easily seen that $(\tilde{X}, \tilde{\mathscr{T}})$ is a compact coherent $T_{0}$-space with a basis of compact open sets.

(d) Let $C$ be a nonempty irreducible closed subset of $(\tilde{X}, \tilde{\mathcal{T}})$. Then $\omega \in C$ and $C=$ $K \cup\{\omega\}$, where $K$ is a closed subset of $(X, \mathscr{T})$. We discuss two cases.

Case 1. $K=\varnothing$. In this case, $C=\{\omega\}$ has a generic point.

Case 2. $K \neq \varnothing$. Necessarily $K$ is an irreducible closed subset of $(X, \mathscr{T})$. Hence there exists $x \in X$ such that $C=\overline{\{x\}}^{X}=\overline{\{x\}}^{\tilde{X}}$.

Therefore, $\tilde{X}$ is a spectral space with a unique closed point $\omega$ (ii) and $\mathrm{X}$ is an open subspace (iv).

(iv) $\Rightarrow$ (iii). Applying Lemma 2.4, $X$ is sober. Let $U$ be an open set of a topological space $X$. If $X$ has a basis $\mathscr{B}$ of compact open sets closed under finite intersections, then $\mathscr{B}_{U}=\{O \in \mathscr{B} \mid O \subseteq U\}$ is a basis of compact open sets of $U$ which is closed under finite intersections, proving that $X$ is a coherent sober space with a basis of compact open sets. 


\section{CSN-spaces}

We divide the proof of our main result into a sequence of lemmas.

Lemma 3.1. Let $X$ be a CSN-space. Then $X$ is coherent and has a basis of compact open sets.

Proof. (1) Let $x \in X$. Since $X$ is a CSN-space, there exists an open set $O_{x}$ and a closed spectral subset $C_{x}$ of $X$ such that $x \in O_{x} \subseteq C_{x}$. Let $\mathscr{B}_{x}$ be a basis of compact open sets of $C_{x}$ and $\mathscr{B O}_{x}=\left\{O \in \mathscr{B}_{x} \mid O \subseteq O_{x}\right\}$. The elements of $\mathscr{B}_{x}$ are compact open sets of $X$. Thus $\mathscr{B}=\bigcup_{x \in X} \mathscr{B} O_{x}$ is a basis of compact open sets of $X$.

(2) To prove that $X$ is coherent, it suffices to show that the intersection of two elements of $\mathscr{B}$ is compact. Let $O_{1}, O_{2}$ be two elements of $\mathscr{B}$. Then there exist $x, y \in X$ such that $O_{1} \in \mathscr{B O}_{x}$ and $O_{2} \in \mathscr{B O}_{y}$. Since $O_{1}$ and $O_{2} \cap C_{x}$ are two compact open sets of $C_{x}, O_{1} \cap$ $\mathrm{O}_{2}=\mathrm{O}_{1} \cap\left(\mathrm{O}_{2} \cap \mathrm{C}_{x}\right)$ is compact.

Lemma 3.2. A space $X$ in which every point has a closed sober neighborhood is sober.

Proof. Let $C$ be a nonempty irreducible closed subset of $X$ and $x \in C$. There exists an open set $O_{x}$ and a closed sober subset $C_{x}$ of $X$ such that $x \in O_{x} \subseteq C_{x}$. Of course, $C=$ $\left(\overline{O_{x}} \cap C\right) \cup\left(\left(X-O_{x}\right) \cap C\right)$ yields $C=\overline{O_{x}} \cap C \subseteq C_{x}$, by irreducibility of $C$. Hence $C$ is an irreducible closed subset of the sober space $C_{x}$. Therefore, $C$ has a generic point.

Since spectral spaces are precisely the coherent compact sober spaces with a base of compact open sets, the following result is an immediate consequence of Lemmas 3.1 and 3.2 .

Corollary 3.3. Let $X$ be a topological space. Then the following statements are equivalent:

(i) $X$ is a spectral space;

(ii) $X$ is a compact CSN-space.

Let $X$ and $Y$ be two topological spaces and $f: X \rightarrow Y$ a continuous map. Hochster [3] has called $f$ spectral if for each compact open set $U$ of $Y, f^{-1}(U)$ is compact in $X$.

We need to recall the patch topology [3]. Let $X$ be a topological space. By the patch topology on $X$, we mean the topology which has as a subbasis for its closed sets the closed sets and compact open sets of the original space. If $X$ has a basis of compact open sets which is closed under finite intersections, then the patch topology has the compact open sets and their complements as an open subbasis. By a patch we mean a closed set in the patch topology. Recall that the patch topology associated to a spectral space is Hausdorff and compact [3]. The set $X$ equipped with the patch topology will be denoted by $X^{\text {patch }}$.

The following remarks are direct consequences of Hochster's results [3].

Remarks 3.4. (1) Let $X$ be a topological space which has a basis of compact open sets closed under finite intersections. Then the following statements are equivalent:

(i) $X$ is spectral;

(ii) $X^{\text {patch }}$ is compact.

(2) Let $X$ be a spectral space and $Y$ a subspace of $X$. Then $Y$ is spectral if and only if $Y$ is a patch in $X$.

(3) If $Y$ is a patch in a spectral space $X$, then $\bar{Y}=\bigcup[\overline{\{y\}} \mid y \in Y]$. 
Lemma 3.5. Let $X$ be a spectral space and $Y$ a topological space which has a basis of compact open sets closed under finite intersections. Let $f: X \rightarrow Y$ be a continuous map. Then the following statements are equivalent:

(i) $f$ is a spectral map;

(ii) $f: X^{\text {patch }} \rightarrow Y^{\text {patch }}$ is continuous.

Proof. (i) $\Rightarrow$ (ii). For each compact open set $U$ of $Y, f^{-1}(U)$ is a compact open set of $X$ and $f^{-1}(Y-U)=X-f^{-1}(U)$ is a complement of a compact open set of $X$. Thus $f: X^{\text {patch }} \rightarrow Y^{\text {patch }}$ is continuous.

(ii) $\Rightarrow(\mathrm{i})$. Let $U$ be a compact open set of $Y$. Then $U$ is closed in $Y^{\text {patch }}$. Hence $f^{-1}(U)$ is a closed subset of the compact space $X^{\text {patch }}$. Thus $f^{-1}(U)$ is compact.

Corollary 3.6. The patch topology of a closed spectral subset of a CSN-space X coincides with the topology induced by the patch topology of $X$.

Proof. Let $C$ be a closed spectral subset of $X$ and $\mathscr{T}$ patch the topology induced by the patch topology of $X$ on $C$. Since $C$ is a closed spectral subset of $X$, the canonical injection $i: C \hookrightarrow$ $X$ is a spectral map. Hence using Lemma $3.5,1_{C}: C^{\text {patch }} \rightarrow\left(C, \mathscr{T}^{\text {patch }}\right)$ is continuous. Now, since $C^{\text {patch }}$ is a compact space and $\left(C, \mathscr{T}^{\text {patch }}\right)$ is Hausdorff, $1_{C}$ is a homeomorphism.

Lemma 3.7. Let $X$ be a CSN-space. Then each closed compact subset of $X$ is a spectral subspace of $X$.

Proof. Let $C$ be a compact closed subset of $X$.

(a) Clearly, $C$ is a $T_{0}$-space, since $X$ is $T_{0}$.

(b) Let $\mathscr{B}$ be a basis of compact open sets of $X$ which is closed under finite intersections. Of course, $\mathscr{B}_{C}=\{U \cap C \mid U \in \mathscr{B}\}$ is a basis of compact open sets of $C$ which is closed under finite intersections.

(c) Finally, one may easily check that any closed subset of a sober space is sober.

Therefore, $C$ is a spectral space.

Corollary 3.8. Let $X$ be a CSN-space. Then for each compact open set $O$ of $X$, there exists a closed spectral subspace $C$ of $X$ such that $O \subseteq C$.

Proof. For each $x \in O$, there exists a closed spectral neighborhood $C_{x}$ of $x$. Hence $O \subseteq$ $\cup\left[C_{x} \mid x \in U\right]$. Since $O$ is compact, there exists a finite subset $M$ of $U$ such that $O \subseteq$ $\bigcup\left[C_{x} \mid x \in M\right]$. Let $C=\bigcup\left[C_{x} \mid x \in M\right]$. Then $C$ is a closed compact subspace of $X$. According to Lemma 3.7, $C$ is a spectral subspace of $X$.

Lemma 3.9. Let $Y$ be a nonempty subset of a CSN-space $X$. Then the following statements are equivalent:

(i) $Y$ is a patch in $X$;

(ii) $Y$ is a CSN-subspace of $X$ and for each compact open set $O$ of $X, O \cap Y$ is compact.

Proof. (ii) $\Rightarrow$ (i). Let $x \in \bar{Y}^{X^{\text {patch }}}$ and $O_{x}$ be a compact open set of $X$ such that $x \in O_{x}$. Then $O_{x} \cap Y$ is a nonempty compact open set of $Y$. According to Corollary 3.8, there exists a closed spectral subspace $C_{x}$ of $Y$ such that $O_{x} \cap Y \subseteq C_{x}$.

Now, remark that the clopen subsets of $X^{\text {patch }}$ form a basis of the patch topology of $X$. 
Let $\mathscr{H}=\left\{(V \cap Y) \cap C_{x} \mid x \in V\right.$ and $V$ is clopen in $\left.X^{\text {patch }}\right\}$. The intersection of any finite family of elements of $\mathscr{H}$ is of the form $(U \cap Y) \cap C_{x}$, where $U$ is clopen in $X^{\text {patch }}$ and $x \in U$. Now, $x \in \bar{Y}^{X^{\text {patch }}}$ implies that $\left(O_{x} \cap Y\right) \cap(U \cap Y) \neq \varnothing$ so that $(U \cap Y) \cap C_{x} \neq \varnothing$. Hence $\mathscr{H}$ has the finite intersection property.

On the one hand, since $C_{x}$ is compact in $Y^{\text {patch }}$, we have $\bigcap[W \mid W \in \mathscr{H}] \neq \varnothing$. On the other hand, $\bigcap\left[V \mid x \in V\right.$ and $V$ is clopen in $\left.X^{\text {patch }}\right]=\{x\}$, since $X^{\text {patch }}$ is a $T_{2}$-space. It follows that $x \in Y$, and thus $\bar{Y}^{X^{\text {patch }}}=Y$. Therefore, $Y$ is a patch in $X$.

(i) $\Rightarrow$ (ii). Let $x \in Y$. Since $X$ is a CSN-space, there exists a closed spectral neighborhood $C_{x}$ of $x$. By Corollary 3.6, $C_{x} \cap Y$ is a patch in $C_{x}$. Hence $C_{x} \cap Y$ is a closed spectral neighborhood of $x$ in $Y$. Thus $Y$ is a CSN-space.

Let $O$ be a compact open set of $X$. Using Corollary 3.8, there exists a closed spectral subspace $C$ of $X$ such that $O \subseteq C$. Since $C \cap Y$ is a patch in $C, C \cap Y$ is a spectral space. According to Corollary 3.6, $O \cap Y=(O \cap Y) \cap C$ is a patch in $C$. It follows that $O \cap Y$ is a compact open set of $Y$.

Corollary 3.10. Let $X$ be a CSN-space and let $Y \subseteq X$. Then the following properties hold.

(1) If $U$ is a compact open set of $X$, then $U$ and $\bar{U}$ are spectral subspaces of $X$.

(2) If $Y$ is a compact subset of $X$, then $\bar{Y}$ is a spectral space.

(3) $Y$ is spectral if and only if $Y$ is a patch compact subset of $X$.

Proof. (1) Let $U$ be a compact open set of $X$; by Corollary 3.8, there exists a closed spectral subset $F$ of $X$ such that $U \subset F$. Hence, as $\bar{U}$ is a closed subspace of the spectral space $F$, it is a spectral subspace of $X$. Now, $U$ is a patch in $\bar{U}$, so that $U$ is spectral.

(2) Since $Y$ is a compact subset of $X$, there exists a compact open set $U$ of $X$ such that $Y \subseteq U$. Hence $\bar{Y}$ is a patch in the spectral space $\bar{U}$ by (1). It follows that $\bar{Y}$ is a spectral space [3].

(3) Suppose that $Y$ is spectral. Then $Y$ is compact. Hence $\bar{Y}$ is spectral by (2). According to M. Hochster, a spectral subspace of a spectral space is necessarily a patch. Thus $Y$ is a patch in $\bar{Y}$. By Corollary 3.6, $Y$ is a patch in $X$.

Conversely, suppose that $Y$ is a compact patch subset of $X$. Then $Y$ is a CSN-subspace of $X$ by Lemma 3.9. Now Corollary 3.3 permits to conclude that $Y$ is spectral.

The following is an immediate consequence of the above corollary.

Corollary 3.11. Let $X$ be a topological space with a basis $\mathscr{B}$ of compact open sets. Then the following statements are equivalent:

(1) $X$ is a CSN-space;

(2) for each $U \in \mathscr{B}, \bar{U}$ is a spectral subspace of $X$.

Now, we are in a position to give a characterization of CSN-spaces.

Theorem 3.12. Let $X$ be a topological space. Then the following statements are equivalent:

(1) $X$ is a CSN-space;

(2) $X$ has the following properties:

(i) $X$ is a coherent sober space with a basis of compact open sets;

(ii) every compact open set $U$ of $X$ has a compact closure (i.e., $U$ is relatively compact). 
Proof. $(1) \Rightarrow(2)$. This follows immediately from Lemmas 3.1 and 3.2 and Corollary 3.10.

$(2) \Rightarrow(1)$. Let $x \in X$. Then there exists a compact open set $O$ of $X$ such that $x \in O$. Property (ii) forces $\bar{O}$ to be a spectral subspace of $X$. Therefore, $X$ is a CSN-space.

Corollary 3.13. Let $X$ be a topological space. Then the following statements are equivalent:

(i) $X$ is a CSN-space;

(ii) $X$ is a locally spectral coherent space in which every compact open set has a compact closure.

Example 3.14. (1) It is easily seen that a spectral space is a CSN-space and a CSN-space is a locally spectral space (see Theorem 3.12).

(2) A locally spectral space need not be a CSN-space. Let $\mathbb{N}$ be the set of integers endowed with the Alexandroff discrete topology $\mathscr{T}$ of the natural order (here the open sets are $\varnothing$ and $\mathbb{N}$, and $\downarrow x=\{y \in \mathbb{N} \mid y \leq x\}$, where $x \in \mathbb{N})$. Clearly, $(\mathbb{N}, \mathscr{T})$ is a noncompact locally spectral space. But $\downarrow 0$ is a compact open set of $(\mathbb{N}, \mathscr{T})$ with closure $\mathbb{N}$. Thus, according to Theorem $3.12,(\mathbb{N}, \mathscr{T})$ is not a CSN-space.

(3) A CSN-space need not be spectral; the same example as in (2) does the job.

\section{Acknowledgment}

The authors gratefully acknowledge helpful comments and suggestions of the anonymous referee.

\section{References}

[1] N. Bourbaki, Éléments de mathématique. Algèbre commutative, Chapitres 1 à 4, Masson, Paris, 1985.

[2] G. Gierz, K. H. Hofmann, K. Keimel, J. D. Lawson, M. W. Mislove, and D. S. Scott, Continuous Lattices and Domains, Encyclopedia of Mathematics and Its Applications, vol. 93, Cambridge University Press, Cambridge, 2003.

[3] M. Hochster, Prime ideal structure in commutative rings, Trans. Amer. Math. Soc. 142 (1969), $43-60$.

[4] K. H. Hofmann and K. Keimel, A general character theory for partially ordered sets and lattices, Mem. Amer. Math. Soc. 122 (1971).

[5] D. S. Scott, Continuous lattices, Toposes, Algebraic Geometry and Logic (Conf., Dalhousie Univ., Halifax, N. S., 1971), Lecture Notes in Math., vol. 274, Springer, Berlin, 1972, pp. 97-136.

Karim Belaid: Département des Mathématiques, École Supérieure des Sciences et Techniques de Tunis, 5 Avenue Taha Hussein, BP 56, Bab Mnara 1008, Tunisia

E-mail address: belaid412@yahoo.fr

Othman Echi: Department of Mathematics, Faculty of Sciences of Tunis, University of Tunis El Manar, Campus Universitaire, 2092 El Manar II, Tunisia

E-mail address: othechi@yahoo.com

Riyadh Gargouri: Department of Mathematics, Institute of Multimedia, Route Mharza Km 1, 5, BP 1030, 3018 Sfax, Tunisia

E-mail address: tn_riadh_30@yahoo.fr 


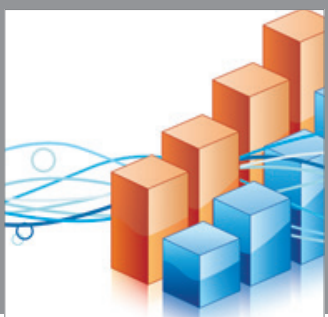

Advances in

Operations Research

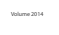

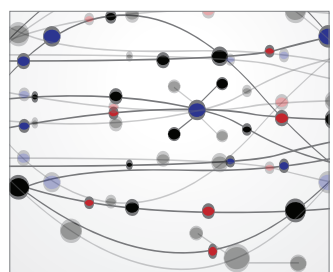

\section{The Scientific} World Journal
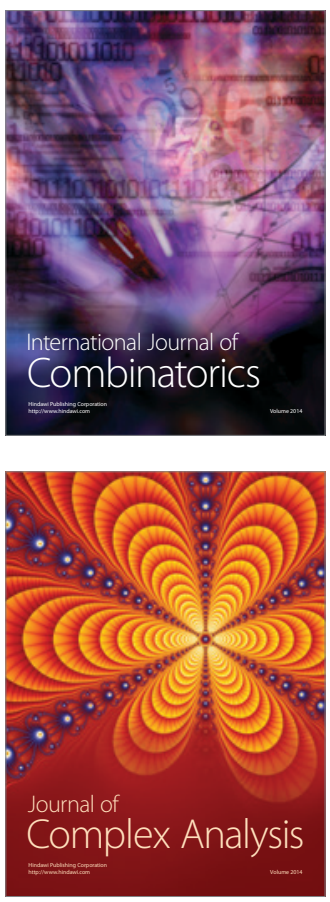

International Journal of

Mathematics and

Mathematical

Sciences
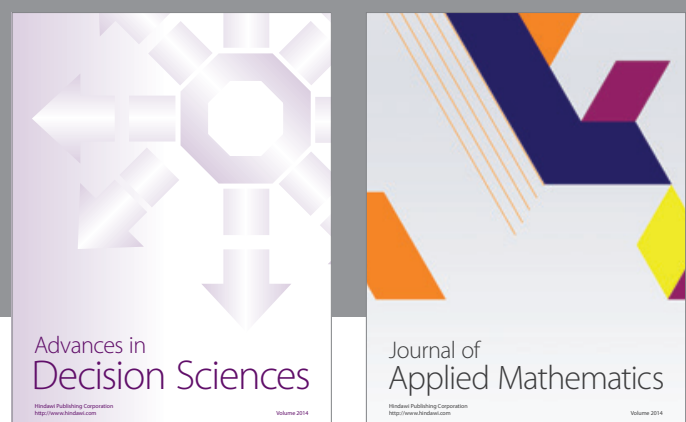

Journal of

Applied Mathematics
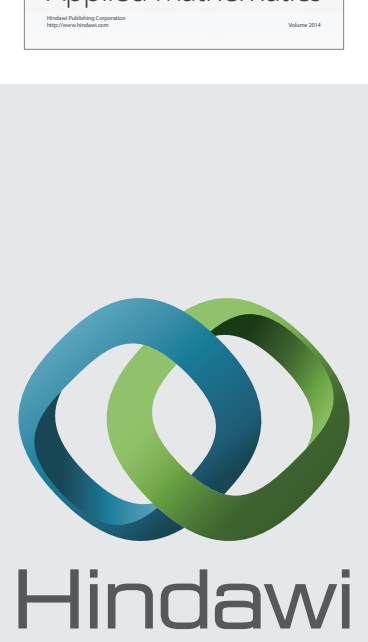

Submit your manuscripts at http://www.hindawi.com
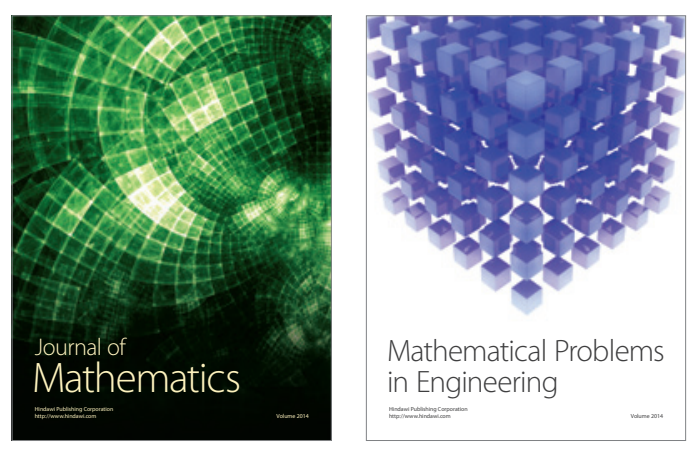

Mathematical Problems in Engineering
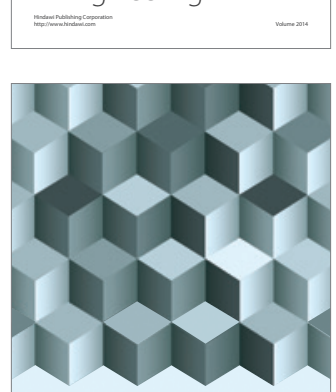

Journal of

Function Spaces
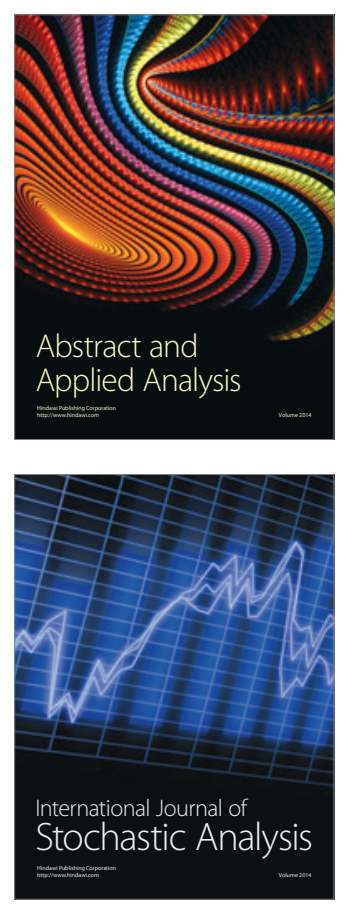

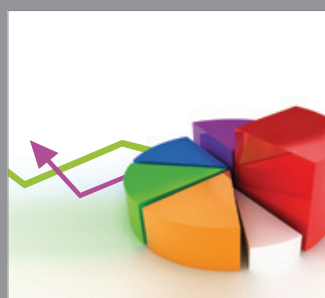

ournal of

Probability and Statistics

Promensencen
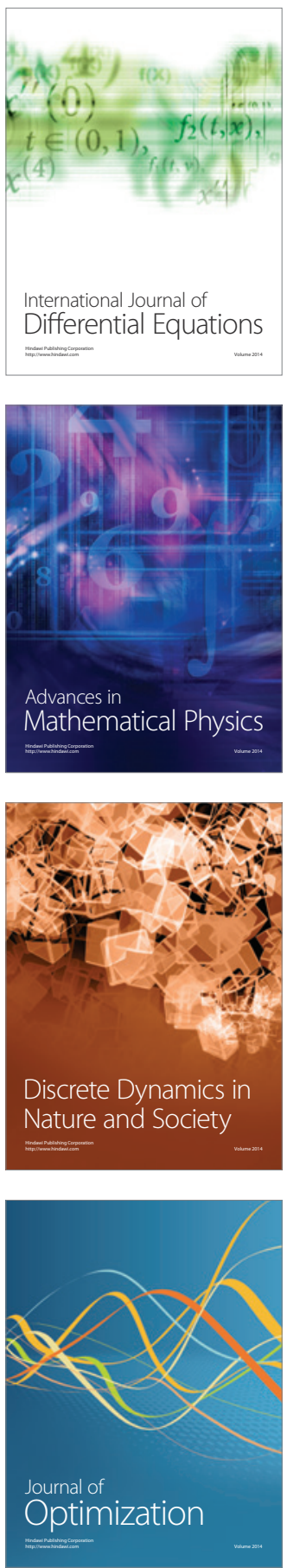